\title{
Compact SOI-Based AWG With Flattened Spectral Response Using a MMI
}

\author{
S. Pathak* , E. Lambert, P. Dumon, D. Van Thourhout and W. Bogaerts \\ *Photonics Research Group (INTEC), Ghent University - imec, \\ Sint-Pietersnieuwstraat 41, B-9000 Ghent, Belgium \\ Email: Shibnath.Pathak@intec.UGent.be
}

\begin{abstract}
We demonstrated an ultra small 12 channels $400 \mathrm{GHz}$ AWG on SOI with flattened spectral response using a MMI mode shaper. Insertion loss and crosstalk are $\mathbf{- 3 . 2 9 d b}$ and $17.0 \mathrm{db}$, respectively. The device size is only $560 \times 350 \mu \mathrm{m}^{2}$.
\end{abstract}

\section{INTRODUCTION}

Arrayed Waveguide Gratings (AWG) are a key component in Wavelength Division Multiplexing (WDM) systems, which form the basis of most modern telecommunication networks. An AWG consists of two free propagation regions (FPR) and an array of waveguides with successive increments in length [5]. In silicon AWGs [2] can be made very compact due to the sharp bend radius [1] and the high group index of the waveguides. However, the optical path length of the array waveguides is several hundreds of wavelengths, which,due to the high index contrast makes the waveguides very sensitive to phase noise. As a result small silicon AWGs typically have higher crosstalk than silica-based and lnP-based AWGs. The spectral response of a conventional AWG is Gaussianlike, but for many WDM applications a flat spectral response is necessary. To achieve this, one of the commonly used methods is the use of a Multi-Mode Interference Coupler (MMI) integrated with the AWG as shown in Figure 1). This method was first adopted by Soole et al. for lnP-based AWGs [6]. In silicon, where the very high contrast makes the design of such MMI couplers and AWGs difficult, AWGs with a flat spectral response have not yet been demonstrated.
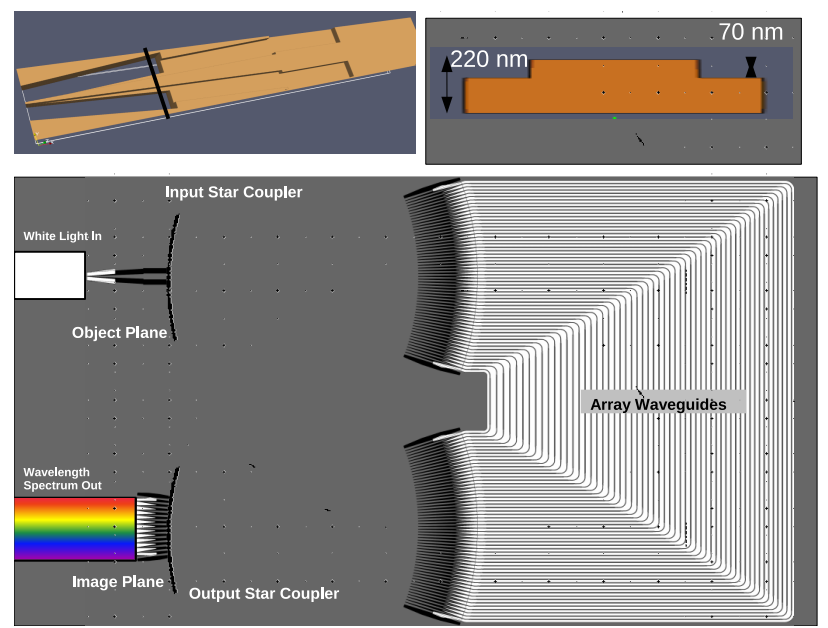

Fig. 1. Schematic diagram of $12 \times 400 \mathrm{GHz}$ MMI-AWG
In this paper we demonstrate compact silicon AWGs with a flat spectral response by integrating a MMI input coupling section. We will also discuss the effect of MMI length on the spectral response of the AWGs.

\section{THEORY AND DESIGN}

Figure 1 shows the schematic layout of an MMI-AWG. The operation of the regular AWG is described as follows. A light beam propagating through the waveguide enters into the first star-coupler and then diverges. This diverging light beam is coupled in the array waveguides and propagates to the second star-coupler. The optical path length difference between two successive waveguides of the array waveguides is $\Delta L=m \cdot \frac{\lambda_{c}}{N_{g}}$, where $m$ is the order of the phased array, $\lambda_{c}$ is the center wavelength of the AWG, and $N_{g}$ is the group index of the waveguide. For this center wavelength the fields from the array waveguides will reach to the second star-coupler with equal phase and the interference will reproduce the field distribution of the object plane at the image plane. With perfect imaging, the field distribution at the image plane will have equal amplitude and phase as the field distribution of the object plane [5]. The phase difference in the array waveguides changes with wavelength because of the length difference and the wavelength-dependence of the propagation constant. This phase delay will introduce a lateral shift of the field profile in the object plane. Thus the spectral response will be determined by the overlap of the field profile at the object plane, with the mode of the waveguides in the image plane combined with the wavelength-dependent shift of the image.

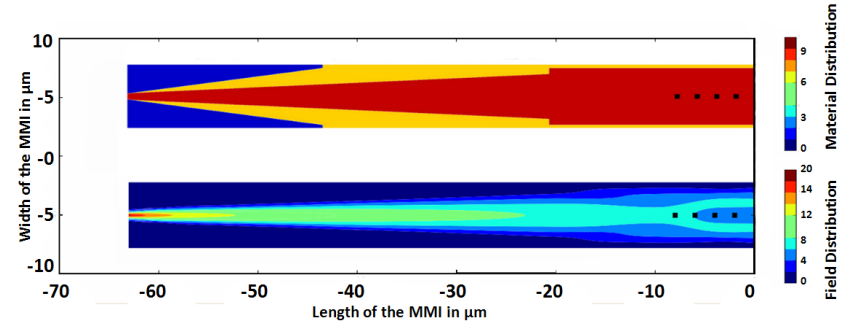

Fig. 2. (Upper) Material geometry of the MMI-aperture use for the CAMFR simulation. (Lower) Simulated field distribution over the MMI-aperture. The black dot indicating the positions $13 \mu \mathrm{m}, 15 \mu \mathrm{m}, 17 \mu \mathrm{m}$ and $19 \mu \mathrm{m}$.

If a MMI is used as the input of the AWG, the image of 
the input field can be tailored. Figure 2 shows the field as it propagates through the MMI. At certain points, we will get a field profile with a flat region in the center, as shown in Figure 3. The overlap between this flat image field and the mode at the image plane with a wavelength-dependent shift will produce a flattened spectral response. The flat region in the spectral response depends on the width of the input field at the end of the MMI. This can be controlled through appropriate choice of the width and length of the MMI. In Figure 3 we can see the field distribution changes from Gaussian-like to a twofold image with a center dip, by changing the length of the MMI from $13 \mu \mathrm{m}$ to $19 \mu \mathrm{m}$, whereby the width of the MMI was fixed to $5 \mu \mathrm{m}$.

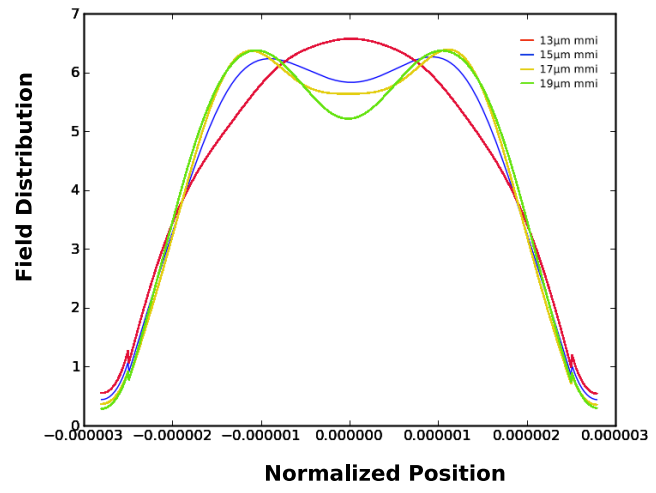

Fig. 3. Field distribution at the end of the MMI for different lengths.

\section{AWG MODEL}

To simulate the AWG we developed a semi-analytical model integrated with our design software. Our simulation model simulates the transmission matrices (T-matrix) of all parts of the AWG using the most suitable method [4]. The AWG is divided in three parts: two star-couplers and the arrayed waveguides. The star couplers themselves are also split in separate parts: the input apertures are simulated numerically using eigenmode propagation, while the free-space propagation is solved analytically using a 2D Fresnel-diffraction scheme. Figure 4(a) illustrates our simulation approach for an AWG.

Let us consider an AWG with $n$ the number of input channels, $m$ the number of output channels and $p$ the number of arms. Then the T-matrix of the input star coupler is $T 1_{n p}$, T-matrix of the output star coupler is $T 3_{m p}$ and T-matrix of the array waveguides is $T 2_{p p}$. So the overall T-matrix $\left(T_{m n}\right)$ of the AWG is equal to $T 3_{m p} \cdot T 2_{p p} \cdot T 1_{n p}^{T}$. For the starcoupler simulation the field-profile of the aperture is simulated by propagating the mode profile of the single-mode input waveguide through the taper and MMI of the input section using an eigenmode solver (CAMFR). Figure 2(upper) shows the material geometry used for the CAMFR simulation. From that simulation result (shown in Figure 2(Lower)) we choose four MMI lengths: $13 \mu \mathrm{m}, 15 \mu \mathrm{m}, 17 \mu \mathrm{m}$ and $19 \mu \mathrm{m}$ (shown

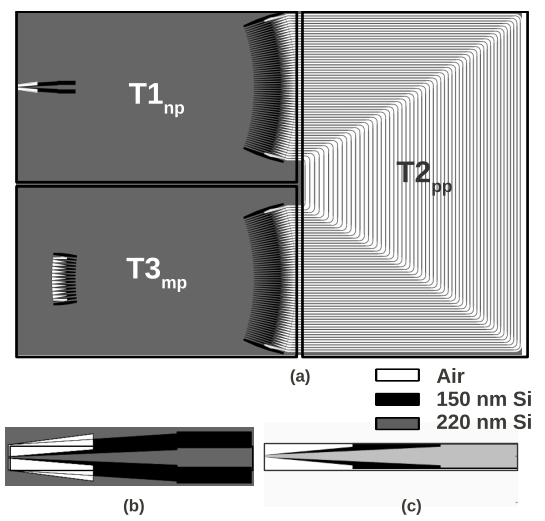

Fig. 4. (a) T-matrix approach for AWG. (b) Virtually fabricated MMI-aperture used in the design. (c) Selected portion of the aperture used for the CAMFR simulation

as black dot in Figure 2) to integrate with a $12 \times 400 \mathrm{GHz}$ AWG. Figure 3 shows the resulting field distributions for the selected MMI lengths. These are then used as input for the analytical simulation of the the free-space propagation region.

\section{RESULTS AND DISCUSSION}

With our simulator we modeled a $12 \times 400 \mathrm{GHz}$ MMIAWG. The MMI is $15 \mu \mathrm{m}$ long and $5 \mu \mathrm{m}$ wide. The simulation result shown in Figure 5) exhibits a central channel loss of $-2.07 d b$ and a nearest channel crosstalk of $19.5 d b$.

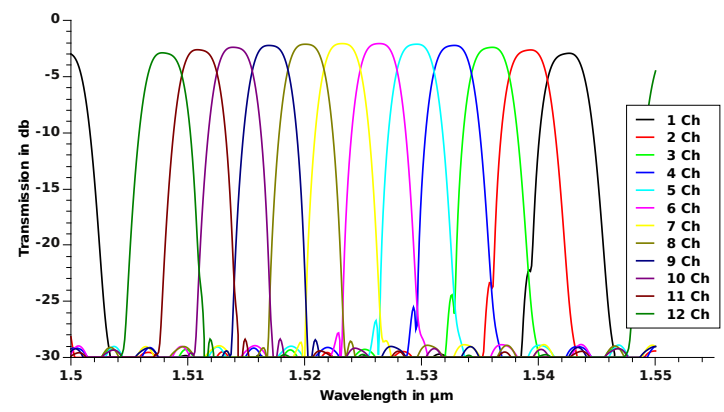

Fig. 5. Simulation Result of $12 \times 400 G H z$ MMI-AWG.

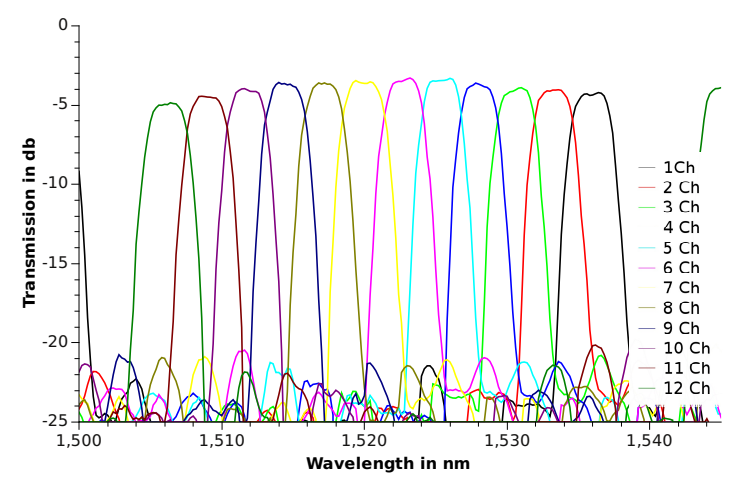

Fig. 6. Measurement Result of $12 \times 400 G H z$ MMI-AWG. 
The experimental result of the same device is shown in Figure 6. The central channel loss for the fabricated device is $-3.29 \mathrm{db}$ and the crosstalk is $17.0 \mathrm{db}$, close to the simulated values. In both simulation and experiment we see that not all channels have a perfect flat-top response: the left and right side channels become slightly asymmetric toward resp. the left and right side. This is the effect of imaging error for outer channels and was described in [3]. The MMI-AWG also has a higher loss than a conventional AWG because of the shape mismatch in the image plane of the AWG and the fact that the power in the image is now spread over a wider area to obtain a flat top wavelength response.

The simulation and experimental spectral responses of all the four chosen MMI lengths $(13 \mu \mathrm{m}, 15 \mu \mathrm{m}, 17 \mu \mathrm{m}$ and $19 \mu \mathrm{m}$ ) are shown in Figure 7 . In both cases we observe that

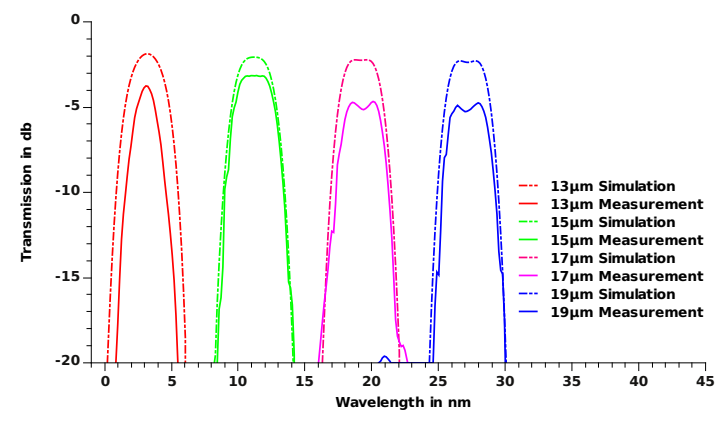

Fig. 7. Simulation result spectral response of $400 \mathrm{GHz}$ AWG with variation of MMI lengths.

the $13 \mu \mathrm{m}$ MMIs have a Gaussian-like response, $15 \mu \mathrm{m}$ MMIs have a flattop response, $17 \mu \mathrm{m}$ MMIs have a small dip at the center of the response and $19 \mu \mathrm{m}$ MMIs have a larger dip. The experimental data for the $17 \mu \mathrm{m}$ and $19 \mu \mathrm{m}$ MMI-AWG show a higher center dip than the simulation results, which can be explained by a small mismatches in MMI width, waveguide thickness or choice of refractive index for the simulation.

\section{CONCLUSION}

An ultra compact twelve channels flattop arrayed waveguide grating(AWG) on silicon-on-insulator(SOI) has been reported. The device size is $560 \times 350 \mu \mathrm{m}^{2}$. Center channel loss is $-3.29 d b$, crosstalk is $17 d b$ and the imaging error is low. The experimental results matches well with our simulation result obtained using our semi-analytical model. The effect of the MMI length on the spectral response of the AWG is investigated in detail through simulation and experimental results.

\section{ACKNOWLEDGMENT}

Part of this work was supported by the European Union in the framework of the FP7 Project ICT-Wadimos. Wim Bogaerts acknowledges the Flemish Research Foundation for a postdoctoral fellowship. The experimental AWG devices were fabricated through ePIXfab.

\section{REFERENCES}

[1] Wim Bogaerts, Pieter Dumon, Dries Van Thourhout, Dirk Taillaert, Patrick Jaenen, Johan Wouters, Stephan Beckx, Vincent Wiaux, and Roel Baets. Compact wavelength-selective functions in silicon-on-insulator photonic wires.

[2] Wim Bogaerts, Shankar Kumar Selvaraja, Pieter Dumon, Joost Brouckaert, Katrien De Vos, Dries Van Thourhout, and Roel Baets. Silicon-oninsulator spectral filters fabricated with cmos technology. IEEE Journal of Selected Topics in Quantum Electronics, 16(1), January 2010.

[3] A. Klekamp and R. Münzer. Calculation of imaging errors of awg. Journal of Lightwave Technology, 21(9), September 2003.

[4] S. Pathak, W. Bogaerts, E. Lambert, P. Dumon, and D. Van Thourhout. Integrated design and simulation tools for silicon photonic arrayed waveguide gratings. Annual Symposium of the IEEE Photononics Benelux Chapter, pages 41-44, November 2010.

[5] Meint K. Smit and Cor van Dam. Phasar-based wdm-devices: Principles, design and applications. IEEE Journal of Selected Topics in Quantum Electronics, 2(2), June 1996.

[6] J.B.D. Soole, M.R. Amersfoort, H.P. Leblanc, N.C. Andreadakis, A. Rajhel, C. Caneau, R. Bhat, M.A. Koza, C. Youtsey, and I. Adesida. Use of multimode interference couplers to broaden the passband of wavelengthdispersive integrated wdm filters. IEEE Photonics Technology Letters, 8(10), October 1996 